\title{
Verbal, Facial and Autonomic Responses to Empathy-Eliciting Film Clips by Disruptive Male Adolescents with High Versus Low Callous-Unemotional Traits
}

\author{
Minet de Wied • Anton van Boxtel • Walter Matthys • \\ Wim Meeus
}

Published online: 26 August 2011

(C) The Author(s) 2011. This article is published with open access at Springerlink.com

\begin{abstract}
This study examined empathy-related responding in male adolescents with disruptive behavior disorder (DBD), high or low on callous-unemotional (CU) traits. Facial electromyographic (EMG) and heart rate (HR) responses were monitored during exposure to empathyinducing film clips portraying sadness, anger or happiness. Self-reports were assessed afterward. In agreement with expectations, DBD adolescents with high CU traits showed significantly lower levels of empathic sadness than healthy controls across all response systems. Between DBD subgroups significant differences emerged at the level of autonomic (not verbal or facial) reactions to sadness, with high $\mathrm{CU}$ respondents showing less HR change from baseline than low $\mathrm{CU}$ respondents. The study also examined basal patterns of autonomic function. Resting HR was not different between groups, but resting respiratory sinus arrhythmia (RSA) was significantly lower in DBD adolescents with high CU traits compared to controls. Results support the notion that $\mathrm{CU}$ traits designate a distinct subgroup of DBD individuals.
\end{abstract}

\footnotetext{
M. de Wied $(\triangle) \cdot$ W. Meeus

Research Centre Adolescent Development, Faculty of Social Sciences, Utrecht University, Heidelberglaan 1, P.O. Box 80140, 3508 TC Utrecht, The Netherlands

e-mail: m.dewied@uu.nl

A. van Boxtel

Department of Psychology, Tilburg University,

P.O. Box 90153, 5000 LE Tilburg, The Netherlands

W. Matthys

Department of Child and Adolescent Psychiatry,

and Rudolf Magnus Institute of Neuroscience,

University Medical Center Utrecht,

Utrecht, The Netherlands
}

Keywords Empathy · Disruptive behavior disorders . Adolescents · Callous-unemotional traits · Facial EMG

\section{Introduction}

Disruptive behavior disorders (DBD), including oppositional defiant disorder (ODD) and conduct disorder (CD), are the most prevalent psychiatric disorders in children and adolescents (e.g., Ford et al. 2003; Verhulst et al. 1997). The Diagnostic and Statistical Manual of Mental Disorders (DSM-IV-TR, American Psychiatric Association 2000) defines ODD as a recurrent pattern of negativistic, defiant, disobedient, and hostile behavior towards authority figures. $\mathrm{CD}$ is a more severe type of disorder, characterized by a repetitive and persistent pattern of behavior in which the basic rights of others or major age-appropriate societal norms or rules are violated. ODD may be a developmental precursor of $\mathrm{CD}$ in late childhood and adolescence, which, in turn, may be a developmental antecedent of antisocial personality disorder (APD) which can be diagnosed from age 18 (APA 2000).

Callous-unemotional (CU) traits identify an important subgroup of antisocial youths in forensic (Vaughn et al. 2008), clinical (Christian et al. 1997), and community samples (Frick et al. 2003, 2005). Antisocial children and adolescents with $\mathrm{CU}$ traits show particular high rates of conduct problems, delinquency and police contacts (see also Frick and White 2008 for a research review). Callousunemotional traits are an extension of the interpersonalaffective dimension of adult psychopathy - a special case of APD - and include a lack of empathy, lack of guilt and low emotional responsiveness (Frick and Hare 2001). Callousunemotional traits in childhood have high genetic loadings (Viding et al. 2005), and are associated with persistent 
criminal behavior (Pardini and Fite 2010) and measures of psychopathy in adulthood (Burke et al. 2007).

In preparation to the fifth edition of the DSM it is proposed to include CU traits as a specifier/subtype for the diagnosis of CD (Frick and Moffit 2010; Scheepers et al. 2011). Although lack of empathy is a defining feature of callous-unemotional traits, surprisingly few studies have directly examined empathy in conduct disordered youths with CU traits. The primary goal of the present study is to examine empathy-related responding in DBD male adolescents with high vs low CU traits and age-matched healthy controls.

Empathy, generally defined as the ability to understand and share another's emotional state (Davis 1996; Hoffman 2008), is a complex multi-component phenomenon, encompassing trait empathy (empathic tendencies of an individual), state empathy (empathic reactions elicited in concrete situations), cognitive empathy (understanding another person's emotions) and affective empathy (sharing another person's emotions). A distinction is often made between different types of affective responding, that is, empathy, sympathy and personal distress (Eisenberg and Eggum 2009). Empathy involves a matching of emotions between the observer and target, that is, feeling with another person. Sympathy consists of feelings of sorrow or concern for the target, thus, feeling for another person. In contrast, personal distress is an aversive reaction, which may consist of feelings of discomfort or anxiety.

Empathy dysfunction proposed for psychopathic individuals is thought to be related to abnormalities in the limbic region, especially the amygdala (Blair 2005, 2007). The amygdala is involved in aversive conditioning and the processing of distress cues, especially fear-related information (Olsson and Phelps 2007). Lesions in this area may lead to a selective impairment in empathy with fear, sadness and possibly disgust (Blair 2007). Recent brain imaging studies show reduced amygdala activation during processing of fearful facial expressions in DBD individuals with high $\mathrm{CU}$ traits relative to healthy controls (Jones et al. 2009; Marsh et al. 2008). Empirical studies on facial affect recognition (Blair et al. 2001; Fairchild et al. 2009; Stevens et al. 2001) further support that children and adolescents with DBD and CU traits have selective impairments in the recognition of fearful and sad expressions (not anger, surprise or happiness). Inverse relationships between CU traits and fear recognition have also been established in studies with community samples of antisocial children and adolescents (Dadds et al. 2006, 2008). Findings from these and other studies (e.g., Dadds et al. 2011) suggest that fear blindness in children with $\mathrm{CU}$ traits is in part due to impaired attention to the eye region of the face, which may also relate to amygdala dysfunction.
Despite the considerable amount of research on facial affect recognition in psychopathic individuals, there has been little attention given to empathy-related responding within more complex empathy-inducing settings. Two studies have recently used self-report measures to examine empathic dysfunction in boys with emotional and behavioral difficulties (Jones et al. 2010), and school-aged children with conduct disorder (AnastassiouHadjicharalambous and Warden 2008a). Both studies demonstrate that those with high CU traits are particularly impaired in affective empathy, but relatively competent in cognitive perspective taking abilities. In an early study, Blair (1999) examined autonomic (electrodermal) responses to distress cue pictures in school-aged boys with emotional and behavioral difficulties. Boys with psychopathic tendencies showed less electrodermal responsiveness than those without these tendencies or healthy controls. Anastassiou-Hadjicharalambous and Warden (2008b) examined verbal and autonomic (heart rate) responses to a videotaped event involving fear in a sample of school-aged children with CD. In line with predictions, high CU children showed less heart rate change than low CU children or controls. Self-reports were not completely consistent with autonomic responses: both $\mathrm{CD}$ groups reported less empathic distress than healthy controls and obtained lower scores on a questionnaire measure of affective empathy. Hence, autonomic response patterns (not self-reports) support distinct empathy deficits across subgroups of children with severe behavior problems. We found no studies replicating these findings in subgroups of DBD adolescents.

The current study adopts a multi-measure approach to examine patterns of affective empathy in DBD adolescents with high vs low CU traits and healthy controls. Respondents were exposed to six empathy-inducing film clips involving either negative (sadness and anger) or positive (happiness) emotions. During exposure, facial electromyographic (EMG) activity in the zygomaticus major (cheek) and corrugator supercilii (eyebrow) muscle regions and heart rate (HR) responses were monitored. After each film clip, respondents were asked about the emotions expressed by the protagonist and the emotions they had experienced themselves.

Facial EMG procedures are commonly used to examine emotional contagion in adults. Positive stimuli, including facial displays of happiness, typically evoke an increase in zygomaticus activity, whereas negative stimuli, including facial displays of sadness and anger, evoke an increase in corrugator activity (e.g., Dimberg 1990; Larsen et al. 2003; Lundqvist and Dimberg 1995). Studies with healthy students show that this typical EMG pattern is less pronounced in low empathizers (Sonnby-Borgström 2002, Sonnby-Borgström et al. 2003; Westbury and Neumann 2008). 
Heart rate responses have often been used to differentiate between sympathy and personal distress (e.g., Eisenberg and Fabes 1990). In normal persons, passive exposure to film clips eliciting sadness, anger, or happiness is generally associated with HR deceleration (e.g., Kreibig et al. 2007; Tsai et al. 2000; Waldstein et al. 2000), possibly reflecting an orienting/attention response. Sympathy or empathic concern is an other-oriented emotion, involving an observer perspective. This state has been associated with HR deceleration. In contrast, personal distress is a self-focused emotion, which has been associated with HR acceleration (Eisenberg and Fabes 1990; Zhou et al. 2003).

While empathy problems proposed for psychopathic individuals may be linked to amygdala dysfunction, empathy problems proposed for the non-psychopathic DBD subtype may stem from multiple sources, including hostility, anxiety and/or emotion regulation dysfunction (e.g., de Wied et al. 2010). Individuals who are emotionally responsive but poor in regulatory skills are at risk to experience personal distress and to become self-focused when witnessing another person in distress (Eisenberg and Eggum 2009). They may show little impairment in euphoric empathy because sharing positive emotions may reduce rather than enhance distress. Recent studies with DBD boys (de Wied et al. 2005, 2009) suggest that those belonging to the more fearful subtype show a selective impairment in empathy with negative (not positive) emotions. Selective impairments in dysphoric (not euphoric) empathy have also been established in studies with antisocial boys from normal populations (Eisenberg et al. 2001; Zhou et al. 2002).

Based on these findings along with findings suggesting that antisocial individuals are weak empathizers (see Lovett and Sheffield 2007; Miller and Eisenberg 1988), we hypothesize that DBD adolescents with low CU traits show less empathy (i.e., report less empathy, show less facial responsiveness and less heart rate reduction) than controls, especially in relation to the film clips involving negative emotions (sadness and anger). Based on theory and research suggesting that those with high $\mathrm{CU}$ traits are selectively impaired in the processing of sadness and fear (e.g., Blair 2007), we hypothesize that DBD adolescents with high CU traits will show a selective impairment in empathic responsivity to the film clips involving sadness (not anger or happiness). Based on evidence suggesting that high CU individuals may lack emotionally responsiveness to negative stimuli (see Frick and White 2008), we hypothesize that DBD adolescents with high CU traits will show stronger impairments in empathic sadness than those with low CU traits.

A secondary goal of the present study is to examine basal autonomic function in DBD adolescents with high and low CU traits and normal controls. Numerous studies show basal autonomic disturbances in children with externalizing disorders, predominantly low resting HR (see Lorber 2004; Ortiz and Raine 2004 for metaanalyses). Low resting HR, thought to be driven by sympathetic underactivation, is one of the best replicated biological markers of aggressive and antisocial behavior (Ortiz and Raine 2004; Raine 2002). Nevertheless, a small number of studies have demonstrated high resting HR in clinic-referred DBD boys (Cole et al. 1996; de Wied et al. 2009; Zahn and Kruesi 1993).

Reduced parasympathetic activation (or cardiac vagal tone) indexed by reduced heart rate variability (HRV) or respiratory sinus arrhythmia (RSA, i.e., the high-frequency component of HRV), has been associated with a variety of psychiatric disorders, including depression, anxiety and aggression (Beauchaine 2001; Thayer and Lane 2000). Reduced cardiac vagal tone has been observed in children and adolescents with DBD (Beauchaine 2001; Beauchaine et al. 2001, 2008; de Wied et al. 2009), also in combination with low sympathetic activation (Beauchaine et al. 2007; Mezzacappa et al. 1997). To identify basal patterns of autonomic activity in the current group of DBD adolescents, resting HR and resting RSA were assessed during a 5-min relaxation video prior to the emotional film clips.

\section{Method}

The Medical Ethical Committee of the University Medical Center Utrecht approved the study protocol, and both parents and adolescents gave written consent prior to participation.

\section{Participants}

DBD male participants, aged between 12 and 15 years, were recruited from special schools for adolescents with severe behavioral problems. Information letters were sent to parents of 89 adolescents. Following written parental consent, a sample of 51 adolescents was screened on the basis of diagnostic information contained in their files at school and information from teachers. The initial screening yielded a sample of 48 adolescents. They were all personally approached for participation in a study on empathic sensitivity. In total, 44 adolescents agreed to participate in the study and signed an informed consent form. IQ was assessed using the Vocabulary and Block Design subtests of the Wechsler Intelligence Scale for Children-Revised for the Netherlands (WISC-RN) (see Wechsler 1974). These subscales have a correlation of 0.90 with the full-scale intelligence quotient (Sattler 1992). The presence of ODD or CD as set out in the DSM-IV-TR (APA 2000) was assessed using the parent version of the Diagnostic Interview Schedule for Children (DISC-IV, 
Dutch version) (Ferdinand and van der Ende 2002). The interviews with parents were carried out by master students in clinical and adolescent psychology. Exclusion criteria for the DBD group included IQ $<80(n=3)$, absence of current $\mathrm{CD} / \mathrm{ODD}$ according to the DISC $(n=10)$, and signs of pervasive developmental disorder $(n=0)$. Of the 31 participants who met the criteria of ODD $(n=17)$ or $\mathrm{CD}(n=14)$, 21 had current comorbid attention deficit hyperactivity disorder (ADHD). Fourteen DBD boys were on psychopharmacological treatment, specifically on methylphenidate $(n=12)$ or risperidone $(n=2)$.

Age-matched normal control (NC) adolescents were recruited from a regular school. Of the 63 parents and children who received an information letter, 38 agreed to participate in the study and completed an informed consent form. Exclusion criteria for the NC group included IQ $<80$ $(n=2)$ or the presence of ODD/CD according to the DISC $(n=3)$. The data of one participant was lost due to procedural errors. Of the 32 healthy controls three had current ADHD, two respondents used methylphenidate.

Parents and teachers of all participants completed the Child Behavior Checklist (CBCL/4-18; Achenbach 1991a) and the Teacher's Report Form (TRF/4-18, Achenbach 1991b), respectively. The CBCL and TRF externalizing (aggressive, rule-breaking) and internalizing (withdrawn, somatic complaints, anxious/depressed) scales were used to confirm the presence of group differences in behavior problems. Parents and teachers also completed the Antisocial Process Screening Device (APSD) (Frick and Hare 2001). The APSD is a 20-item questionnaire designed to measure psychopathic traits in children and adolescents. The scale includes three factors: Callous-Unemotional (six items), Narcissism (seven items), and Impulsivity (five items). Because Narcissism and Impulsivity were highly correlated in our study $(r=0.83, p<0.001)$ they were combined to form the I/CP factor, reflecting impulsivity and conduct problems (Frick and Hare 2001). Ratings from parents and teachers $\left(r_{\mathrm{CU}}=0.46, p<0.001 ; r_{\mathrm{I} / \mathrm{CP}}=0.57, p<\right.$ 0.001 ) were combined by using the highest item score for each item (see Frick and Hare 2001). Internal consistency was acceptable for the $\mathrm{CU}$ dimension $(\alpha=0.71)$, and good for the $\mathrm{I} / \mathrm{CP}$ dimension $(\alpha=0.93)$.

The CU dimension was used to assign participants to groups. The CU scale, including items as "does not show emotions" or "feels bad or guilty (reversed scored)", captures the callous interpersonal style that is critical to the construct of psychopathy. DBD adolescents with a raw score of 9 and above on the $\mathrm{CU}$ dimension were assigned to the $\mathrm{DBD} / \mathrm{CU}+$ group $(n=14)$. A raw score of 9 on $\mathrm{CU}$ converts to a $T$-score of 67 , which is high enough to be considered clinically significant. Those with lower scores were assigned to the $\mathrm{DBD} / \mathrm{CU}$ - group $(n=17)$. The high $\mathrm{CU}$ group included equal numbers of $\operatorname{ODD}(n=7)$ and $\mathrm{CD}$ $(n=7)$, the low CU group included more ODD $(n=10)$ than CD $(n=7)$. The high CU group included $64 \%$ comorbid ADHD respondents, the low CU group 71\%. Respondents using methylphenidate were equally divided among the high (36\%) and low (41\%) CU groups. All adolescents in the control group obtained $\mathrm{CU}$ scores in the normal range (raw scores $\leq 6, T$-scores $\leq 55$ ).

Descriptive characteristics of the samples are included in Table 1. One-way analyses of variance (ANOVAs) were conducted to examine differences among groups. The analyses revealed no age differences, nor differences in intelligence across the three groups. The analyses demonstrated expected differences between both DBD groups and healthy controls on the CBCL and TRF externalizing, aggressive and rule-breaking scales, such that DBD adolescents obtained higher scores than controls. The analyses also revealed significant differences between both DBD groups and healthy controls on the CBCL and TRF internalizing, withdrawn and anxious/depressed scales, with higher scores for DBD adolescents than controls.

Additional $t$-tests according to Fisher's LSD method confirmed expected differences in CU traits (see Table 1). The high CU group (DBD/CU+) obtained significantly higher scores than the low $\mathrm{CU}$ group (DBD/CU-) and controls on the APSD total list score and both the CU and I/ $\mathrm{CP}$ dimensions. The $\mathrm{DBD} / \mathrm{CU}$ - group, in turn, obtained higher scores than controls on the APSD total list score and $\mathrm{I} / \mathrm{CP}$ dimension, not on the $\mathrm{CU}$ dimension. In agreement with studies showing that $\mathrm{CU}$ traits relate to high levels of aggression/delinquency (e.g., Frick and White 2008), the $\mathrm{DBD} / \mathrm{CU}+$ group obtained significantly higher scores than the DBD/CU- group on the CBCL externalizing scale, and on both the CBCL and TRF aggressive and rule-breaking scales. Findings support the hypothesis that $\mathrm{CU}$ traits identify a more severe subgroup of DBD adolescents.

\section{Materials}

Relaxation Video A 5-min excerpt from an aquatic video (Coral Sea Dreaming, Small World Music Inc.) was used to assess resting levels of RSA and HR. The same aquatic video has been used in a study conducted by Piferi et al. (2000), who demonstrated that watching an excerpt from this relaxing video is more effective in lowering cardiovascular activity levels than resting quietly. To ensure recovery from emotional arousal induced by the film clips, participants also viewed 1-min excerpts from the same aquatic video prior to each emotional film clip.

Emotional Film Clips Six film clips were used to evoke empathic reactions: two sadness clips involving a girl sent to boarding school and a boy who fails at selection training for a soccer tournament, two anger clips involving a girl 
Table 1 Characteristics of boys with disruptive behavior disorders (DBD) and normal controls (NC)

\begin{tabular}{|c|c|c|c|c|c|c|c|}
\hline & \multicolumn{2}{|c|}{$\mathrm{NC}(n=32)$} & \multicolumn{2}{|c|}{$\mathrm{DBD} / \mathrm{CU}-(n=17)$} & \multicolumn{2}{|c|}{$\mathrm{DBD} / \mathrm{CU}+(n=14)$} & \multirow[b]{2}{*}{$F$-values } \\
\hline & M & $S D$ & $M$ & $S D$ & $M$ & $S D$ & \\
\hline \multicolumn{8}{|l|}{ APSD } \\
\hline Total list score & $12.91^{\mathrm{a}}$ & 5.20 & $24.71^{\mathrm{b}}$ & 5.99 & $32.14^{\mathrm{c}}$ & 3.30 & $78.05 * * *$ \\
\hline CU dimension & $5.75^{\mathrm{a}}$ & 2.83 & $6.82^{\mathrm{a}}$ & 1.18 & $9.86^{\mathrm{b}}$ & 0.95 & $17.51 * * *$ \\
\hline $\mathrm{I} / \mathrm{CP}$ dimension & $3.28^{\mathrm{a}}$ & 1.81 & $8.00^{\mathrm{b}}$ & 2.38 & $9.61^{\mathrm{c}}$ & 1.30 & $68.04 * * *$ \\
\hline \multicolumn{8}{|c|}{ Child Behavior Checklist } \\
\hline Externalizing & $47.59^{\mathrm{a}}$ & 8.24 & $68.41^{\mathrm{b}}$ & 3.97 & $75.07^{\mathrm{c}}$ & 7.90 & $87.85^{* * *}$ \\
\hline Aggressive & $52.81^{\mathrm{a}}$ & 4.34 & $68.71^{\mathrm{b}}$ & 6.28 & $77.86^{\mathrm{c}}$ & 11.04 & $74.60 * * *$ \\
\hline Rule-breaking & $52.19^{\mathrm{a}}$ & 2.96 & $66.12^{\mathrm{b}}$ & 4.37 & $71.86^{\mathrm{c}}$ & 8.83 & $85.85 * * *$ \\
\hline Internalizing & $49.63^{\mathrm{a}}$ & 9.53 & $61.76^{\mathrm{b}}$ & 7.81 & $62.07^{\mathrm{b}}$ & 9.23 & $14.54 * * *$ \\
\hline Withdrawn & $54.81^{\mathrm{a}}$ & 4.68 & $61.94^{\mathrm{b}}$ & 6.40 & $66.21^{\mathrm{b}}$ & 9.51 & $17.12 * * *$ \\
\hline Somatic Complaints & 55.06 & 7.44 & 59.47 & 7.97 & 58.21 & 8.13 & 2.04 \\
\hline Anxious/Depressed & $52.56^{\mathrm{a}}$ & 4.67 & $59.82^{\mathrm{b}}$ & 9.33 & $59.57^{\mathrm{b}}$ & 8.03 & $8.31^{* *}$ \\
\hline \multicolumn{8}{|l|}{ Teacher's Report Form } \\
\hline Externalizing & $48.06^{\mathrm{a}}$ & 8.41 & $66.82^{\mathrm{b}}$ & 9.24 & $72.00^{\mathrm{b}}$ & 6.39 & $52.20 * * *$ \\
\hline Aggressive & $53.31^{\mathrm{a}}$ & 5.29 & $67.47^{\mathrm{b}}$ & 11.09 & $73.50^{\mathrm{c}}$ & 9.85 & $34.89 * * *$ \\
\hline Rule-breaking & $52.16^{\mathrm{a}}$ & 4.29 & $65.24^{\mathrm{b}}$ & 9.24 & $70.29^{c}$ & 7.68 & $41.51 * * *$ \\
\hline Internalizing & $44.16^{\mathrm{a}}$ & 6.24 & $61.29^{\mathrm{b}}$ & 8.00 & $59.86^{\mathrm{b}}$ & 5.61 & $48.84 * * *$ \\
\hline Withdrawn & $51.41^{\mathrm{a}}$ & 2.60 & $58.12^{\mathrm{b}}$ & 6.03 & $60.71^{\mathrm{b}}$ & 3.43 & $32.74 * * *$ \\
\hline Somatic Complaints & $50.38^{\mathrm{a}}$ & 2.12 & $58.06^{\mathrm{b}}$ & 8.96 & $55.86^{\mathrm{b}}$ & 8.25 & $9.65 * * *$ \\
\hline Anxious/Depressed & $51.03^{\mathrm{a}}$ & 1.81 & $62.47^{\mathrm{b}}$ & 8.11 & $57.86^{\mathrm{c}}$ & 5.80 & $29.07 * * *$ \\
\hline Resting RSA proportional & $0.47^{\mathrm{a}}$ & 0.16 & $0.46^{\mathrm{a}}$ & 0.14 & $0.35^{\mathrm{b}}$ & 0.15 & $3.20 *$ \\
\hline Resting $\mathrm{RSA}_{\text {log power }}$ & $7.84^{\mathrm{a}}$ & 0.86 & $7.80^{\mathrm{a}}$ & 0.68 & $6.90^{\mathrm{b}}$ & 1.30 & $3.22 *$ \\
\hline Resting HR & 71.18 & 9.28 & 75.39 & 12.58 & 75.68 & 13.61 & 1.17 \\
\hline Age & 13.75 & 0.76 & 13.29 & 0.85 & 13.93 & 1.07 & 2.39 \\
\hline IQ & 99.09 & 9.11 & 97.65 & 10.34 & 95.43 & 9.34 & 0.73 \\
\hline
\end{tabular}

$\mathrm{RSA}_{\text {proportional }}=$ respiratory sinus arrhythmia (power within RSA frequency band as a proportion of power within entire frequency range of HRV spectrum); $\mathrm{RSA}_{\log \text { power }}=$ respiratory sinus arrhythmia (natural logarithm of power within RSA frequency band); HR = heart rate (beats per minute). Means with different superscripts are significantly different as revealed by $t$-tests $(p \leq 0.05)$

$* * * p<0.001 ; * * p<0.01 ; * p<0.05$

under house arrest and a boy being hindered in an important cycle race, and two happiness clips involving a girl passing her finals, and a boy winning a song contest. The clips were assembled from documentary films (broadcast by the Dutch public broadcasting companies) and pretested in a pilot study. Male $(n=25)$ and female $(n=25)$ adolescents, aged between 12 and 15 years, were exposed to eleven pilot film clips. They were asked to identify the emotions conveyed by the story character and to rate their empathic feelings. The clips selected for use in the current study all represent one prominent emotion (sadness, anger or happiness), agreed upon by more than $95 \%$ of the male and female adolescents.

Repeated measures ANOVAs further revealed that the film clips evoke distinct emotions. Both male and female adolescents reported significantly higher levels of empathic sadness during the sadness clips than during the anger $(F(1$, $\left.48)=63.45, \quad p<0.001, \eta_{\mathrm{p}}^{2}=0.57\right)$ and happiness clips
$\left(\mathrm{F}(1,48)=77.26, p<0.001, \eta_{\mathrm{p}}^{2}=0.62\right)$. Significantly higher levels of empathic happiness were reported during the happiness clips than during the sadness $(F(1,48)=101.64$, $\left.p<0.001, \eta_{\mathrm{p}}^{2}=0.68\right)$ and anger clips $(F(1,48)=80.07, p<$ $\left.0.001, \eta_{\mathrm{p}}^{2}=0.63\right)$. Significantly higher levels of empathic anger were reported during the anger clips than during the happiness clips $\left(F(1,48)=36.04, p<0.001, \eta_{\mathrm{p}}^{2}=0.43\right)$, though anger and sadness clips evoked equal levels of empathic anger $\left(F(1,48)=2.18, p=0.15, \eta_{\mathrm{p}}^{2}=0.04\right)$.

The film clips varied in length between 124 and $157 \mathrm{~s}$. A voice-over sketching the situation (edited into the title description) introduced each clip. The closing scene in which the story character portrayed intense facial and vocal expressions of emotion were taken as target episode. The targets varied in length between 24 and $56 \mathrm{~s}$. Heart rate and facial EMG responses were analyzed during the target 
episodes and during the first $10 \mathrm{~s}$ of the opening scene, which served as baseline.

Apparatus and Stimulus Presentation Participants were individually tested in a laboratory room at the university. The room contained a testing area and observation unit partitioned by a one-way screen. Both units were equipped with a personal computer, one for stimulus presentation (in the test room) and one for online control of data collection (in the observation unit). Both computers were connected to a small portable digital recorder for the preprocessing and storage of physiological data that was attached to the participant's chair (Vitaport II, TEMEC Instruments B.V., Kerkrade, The Netherlands).

All film clips were digitized and presented (in randomized order) on a 17-inch computer screen (Philips 109E50). A program written with Delphi 6 was used to control stimulus presentation and to collect children's self-reports. The program also generated signals marking onset and offset of each film clip which were stored on the Vitaport II recorder.

\section{Response Measures}

Self-report Empathy After each film clip the participant was asked to identify the quality and rate the intensity of emotions portrayed by the protagonist and their own experienced emotions. Six cartoon-faces emerged on the computer screen representing the facial expression of sadness, happiness, anger, fear, surprise and a neutral face. Identification of the quality of emotion was established by marking one or more faces through a mouse click. When the participant marked an emotional face, he was asked to rate the intensity of the particular emotion. Responses indicating that the participant observed a particular emotion during the target scene and experienced that same emotion (affect match) were counted empathic responses. When more than one emotion was reported, the most prominent and strongest one was reckoned. Empathic responses were rated on a 5 -point scale $(0=$ no empathy, $4=$ maximal response).

Physiological Recordings Bipolar EMG recordings were made from the left zygomaticus and corrugator muscles using surface $\mathrm{Ag} / \mathrm{AgCl}$ electrodes (contact area $2 \mathrm{~mm}$ diameter; $15 \mathrm{~mm}$ distance between electrode centers). The electrocardiogram (ECG) was recorded with electrodes on the chest (sternum-V6 lead). EMG and ECG signals were antialiasing filtered $(512 \mathrm{~Hz}$ lowpass filter) and digitized at a rate of 1,024 Hz. EMG signals were digitally filtered offline to remove low-frequency artifacts (20 Hz highpass filter) and any influence of $50-\mathrm{Hz}$ power line interference $(48-52 \mathrm{~Hz}$ bandreject filter). The ECG was digitally bandpass filtered $(5-30 \mathrm{~Hz})$ to suppress baseline shifts, exceptionally large T-waves, and high-frequency artifacts such as EMG potentials. Data were then visually inspected for remaining artifacts. An automatic procedure was executed offline to detect ECG R-waves and to make corrections for (a) prolonged heart periods due to missing R-waves and (b) short heart periods due to false R-waves (for details of this procedure, see de Wied et al. 2009).

Mean HR and mean rectified EMG amplitudes were determined during the baseline and target periods of each film clip. Resting HR and resting RSA were calculated during the 300-s presentation of the aquatic film clip. RSA was determined by calculating the power spectrum of the heart periods in the $0.15-0.40 \mathrm{~Hz}$ frequency band using the computer program CARSPAN for Windows 1.34 (Mulder et al. 2007; for a detailed account of this procedure see de Wied et al. 2009). Power spectral estimates were normalized by expressing them as squared coefficients of variation which has as an effect that the power spectrum becomes independent of the mean heart period during the analysis interval. Since total HRV power may widely vary between individuals due to constitutional differences, power in the RSA band was additionally normalized by expressing it as a proportion of total power within the entire frequency range of the HRV spectrum (i.e., the $0.02-0.40 \mathrm{~Hz}$ frequency band) as recommended by the Task Force of the European Society of Cardiology and the North American Society of Pacing and Electrophysiology (1996). We also quantified resting RSA as the natural logarithm of the spectral power in the $0.15-0.40 \mathrm{~Hz}$ frequency band to enable a better comparison with the results of earlier studies assessing resting RSA levels in DBD children or adolescents which applied this quantification method.

The power in the RSA frequency band is believed to be regulated predominantly by the parasympathetic branch of the autonomic nervous system (Berntson et al. 1997). When estimating interindividual differences in parasympathetic cardiac activation based on RSA, a correction has to be made for confounding effects of individual differences in respiratory parameters (Grossman and Kollai 1993; Grossman and Taylor 2007). During a quiet state, such as when viewing the aquatic film clip, it may be considered sufficient to control for group differences in respiratory frequency (Ritz and Dahme 2006). As we did not record respiration, we derived the peak respiratory frequency during the 300-s aquatic film clip from the peak frequency of the RSA power spectrum (Denver et al. 
2007; Thayer et al. 2002) and compared it between the DBD groups and the control group.

\section{Procedure}

Participants were seen individually by two female experimenters. The experimenters were trained and used a written protocol detailing the verbal instructions, electrode placement and working of the computerized empathy task. All participants were told that they would view six short film clips about which several questions would be asked. They were also told that physiological recordings would be made to examine bodily reactions during film exposure. Prior to the test session, all participants completed the two WISC-RN subtests in the test room. Next, the electrodes were attached and the participant was seated in an armchair facing the monitor of the stimulus computer at approximately $80 \mathrm{~cm}$ distance. The lights were dimmed and the participant was instructed to watch a 5 min relaxation video. After that, the experimenter demonstrated the computerized empathy task. Once familiar with the setting and procedure, the test session was started, which took about $45 \mathrm{~min}$ to complete. During vignette presentation the experimenter remained in the observation unit. Upon completion, the participant was paid 20 euros for participation.

\section{Statistical Analyses}

Mean EMG amplitude during the target scene was expressed as a percentage of the mean amplitude during the baseline period. The HR response was expressed as the difference score between mean HR during the target and baseline period. The HR and EMG scores were averaged across the two sadness, anger and happiness clips, generating three HR scores, three corrugator EMG and three zygomaticus EMG scores for each individual. The empathy ratings were also averaged across the clips, generating three self-report scores: empathic sadness, anger and happiness.

Based on the literature suggesting that antisocial behavior relates differently to dysphoric vs euphoric empathy (e.g., de Wied et al. 2010), and studies suggesting that children with psychopathic tendencies are selectively impaired in the processing of distress cues (e.g., Blair 1999), empathy-related response patterns were examined separately for each emotion. ANOVAs and follow-up $t$-tests were used to examine differences between DBD subgroups and controls. Probabilities of all tests were two-tailed, unless the direction of findings was predicted in which case it is explicitly mentioned that one- tailed testing was performed. A significance level of 0.05 was adopted in all tests.

\section{Results}

Medication Checks

Prior to conducting the main analyses of the study we compared DBD subjects who were using methylphenidate with those who were not on cardiac output at rest. Independent samples $t$-tests did not reveal a significant difference for resting HR, $t(29)=-1.38, p=0.18, d=0.48$, nor for resting RSA, $t(29)=1.16, p=0.26, d=0.39$. Also, we compared DBD subjects who were using methylphenidate with those who were not on all empathy-related responses. Except for a marginal difference in corrugator EMG responses to sadness, $t(29)=-1.74, p=0.092, d=0.61$, with those on medication showing a stronger increase in corrugator EMG activity than those who were not, and a marginal difference in HR responses to anger, $t(29)=-1.74$, $p=0.092, d=0.66$, with those on medication showing less HR deceleration (more HR acceleration) than those who were not, no significant differences emerged on any measure (all $p$ 's $>0.1$ ). Therefore, medication (yes/no) was included as a covariate in the statistical analyses of both corrugator EMG responses to sadness and HR responses to anger to correct for possible influences of methylphenidate. The scores of treated and untreated DBD subjects were collapsed in all other statistical analyses.

\section{Validity Checks}

To check whether our stimulus material evoked classic EMG response patterns, repeated measures ANOVAs were conducted with group (DBD vs NC) as the betweensubjects factor and film clips (positive vs negative) as the within-subjects factor. Medication was included as a covariate in a repeated measures analysis of covariance (ANCOVA) conducted for corrugator EMG responses to sadness. Consistent with the literature (Larsen et al. 2003), the positive film clips evoked in both groups a stronger increase in zygomaticus EMG activity than the negative clips, $F_{\text {happiness vs sadness }}(1,61)=18.91, p<0.001$, $\eta_{\mathrm{p}}^{2}=0.24 ; F_{\text {happiness }}$ vs anger $(1,61)=15.95, p<0.001$, $\eta_{\mathrm{p}}^{2}=0.21$. Vice versa, the negative film clips evoked in both groups a stronger increase in corrugator EMG reactivity than the positive clips, $F_{\text {sadness vs happiness }}(1,60)=36.03, p<$ $0.001, \eta_{\mathrm{p}}^{2}=0.38 ; F_{\text {anger vs happiness }}(1,61)=100.80, p<0.001$, $\eta_{\mathrm{p}}^{2}=0.62$. These findings highlight the validity of the corrugator EMG response to reflect expressions of negative 
emotions, and the zygomaticus EMG response to reflect expressions of positive emotions.

All participants $(100 \%)$ correctly identified the prominent emotion exhibited by the protagonist, that is, sadness in the sadness clips, anger in the anger clips and happiness in the happiness clips. Thus potential differences between groups in measures of affective empathy cannot be attributed to differences in emotion recognition.

\section{Group Differences in Empathy-Related Responses}

Self-reports ANOVAs yielded significant group differences in empathic sadness, $F(2,60)=3.81, p=0.028, \eta_{\mathrm{p}}^{2}=0.11$, and happiness, $F(2,60)=6.84, p=0.002, \eta_{\mathrm{p}}^{2}=0.19$, not in empathic anger, $F(2,60)=1.78, p=0.177, \eta_{\mathrm{p}}^{2}=0.06$. Consistent with expectations, follow-up $t$-tests revealed that the $\mathrm{DBD} / \mathrm{CU}+$ group reported significantly less empathic sadness than the control group, $t(44)=2.64$, $p=0.006$, one-tailed, $d=0.98$. As shown in Fig. 1a, the high CU group reported less empathy than the low CU group and the low CU group, in turn, reported less empathic sadness than the control group, but the analyses did not reach significance: $t(29)=1.43, p=0.081$, one-tailed, $d=0.55, t(47)=1.39, p=0.085$, one-tailed, $d=0.43$, respectively. As to happiness, follow-up $t$-tests revealed that both $\mathrm{DBD} / \mathrm{CU}+$ and $\mathrm{DBD} / \mathrm{CU}$ - reported significantly less empathic happiness than controls: $t(44)=3.12, p=0.003$, $d=1.13, t(47)=2.42, p=0.020, d=0.77$, respectively. No significant difference emerged between DBD subgroups, $t(29)=1.05, p=0.302, d=0.38$.

Zygomaticus EMG The ANOVA yielded a marginally significant difference between groups in zygomaticus EMG reactivity during happiness, $F(2,60)=3.11, p=$ 0.052, $\eta_{\mathrm{p}}^{2}=0.09$. Follow-up $t$-tests demonstrated that the $\mathrm{DBD} / \mathrm{CU}$ - group showed a significantly smaller increase in zygomaticus EMG activity during happiness than controls, $t(47)=2.13, p=0.038, d=0.73$ (see Fig. 1b). No significant difference emerged between $\mathrm{DBD} / \mathrm{CU}+$ and controls, $t(44)=1.37, p=0.176, d=0.50$, nor between DBD subgroups, $t(29)=-1.30, p=0.205, d=0.46$. Differences between groups in zygomaticus EMG responses during sadness, $F(2,60)=0.95, p=0.394, \eta_{\mathrm{p}}^{2}=0.03$, and anger, $F(2,60)=2.95, p=0.060, \eta_{\mathrm{p}}^{2}=0.09$, were not predicted, nor observed. Interestingly, however, the high $\mathrm{CU}$ group showed a significant increase in zygomaticus EMG activity during the anger clips relative to the low $\mathrm{CU}$ group, $t(29)=-2.24, p=0.033, d=0.80$, suggesting that they felt amused rather than angered. Increased zygomaticus activity while processing anger cues (suggesting laughing) has been observed more often among low empathizers in the normal population (Sonnby-Borgström 2002). a

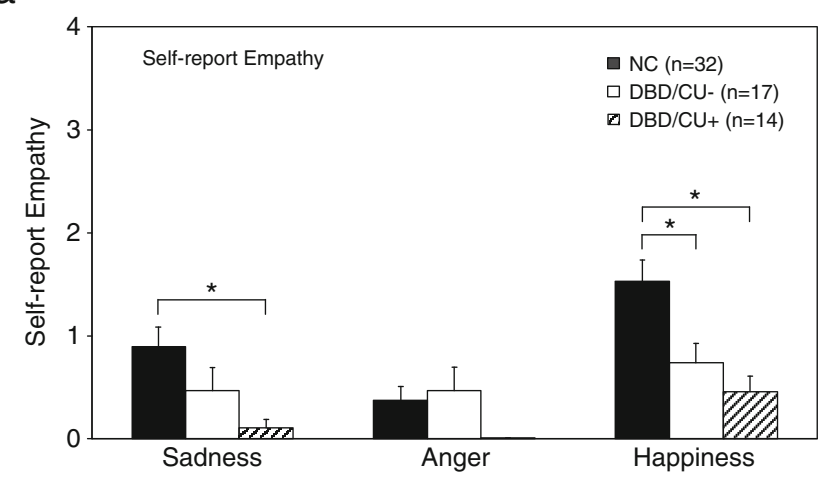

b
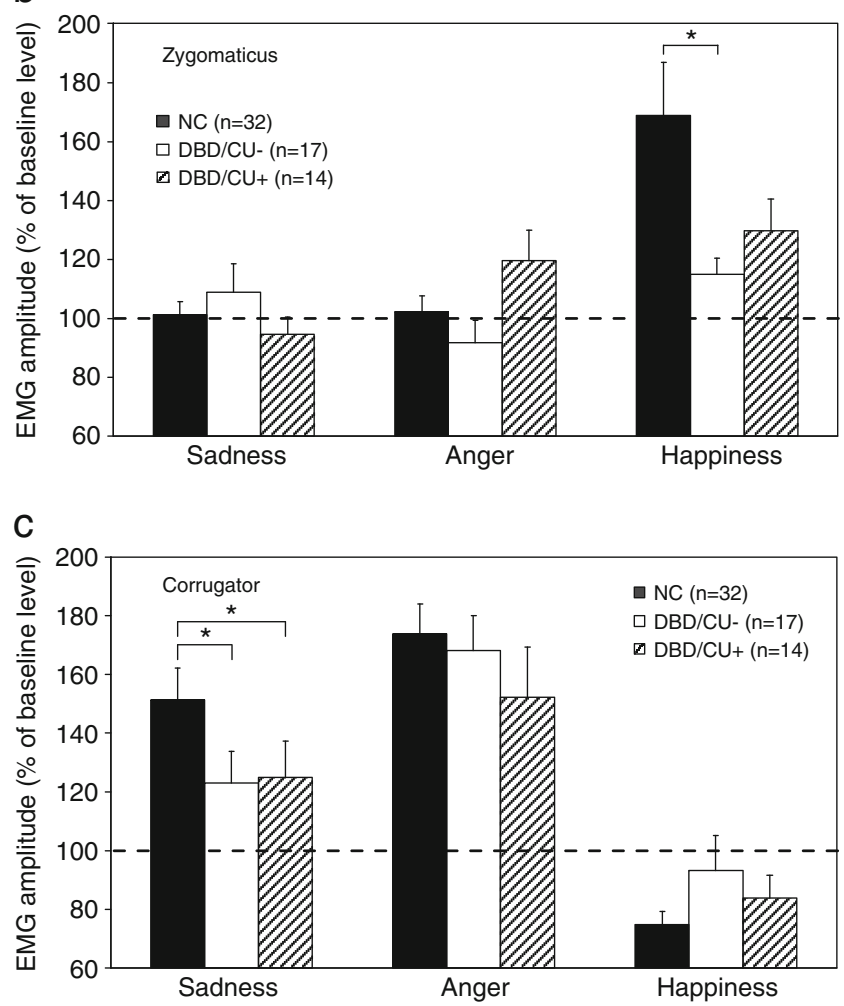

d

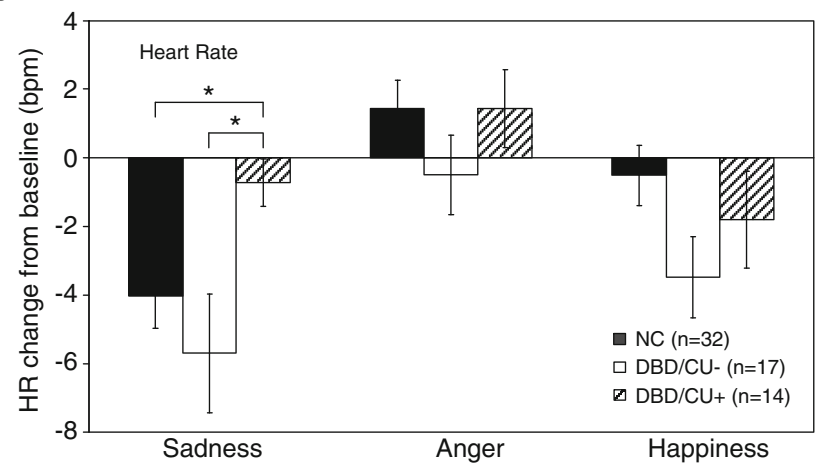

Fig 1 Mean (+SE) self-report empathy (a), zygomaticus EMG (b), corrugator EMG (c), and HR reactivity (d), across film clips for DBD adolescents with high (DBD/CU+), versus low CU traits (DBD/CU-), and healthy controls (NC) 
Corrugator EMG ANOVAs yielded no significant differences between groups in corrugator EMG responses during anger, $F(2,60)=0.69, p=0.50, \eta_{\mathrm{p}}^{2}=0.02$, and happiness, $F$ $(2,60)=1.73, p=0.186, \eta_{\mathrm{p}}^{2}=0.06$. An ANCOVA, with medication as a covariate, revealed a significant difference between groups in corrugator EMG responses during sadness, $F(2,59)=4.19, p=0.020, \eta_{\mathrm{p}}^{2}=0.12$ (see Fig. 1c). In agreement with expectations, pairwise comparisons revealed that both DBD subgroups showed a smaller increase in corrugator EMG activity than healthy controls: contrast estimate $(\mathrm{DBD} / \mathrm{CU}+\mathrm{vs}$ controls $)=38.94, p=0.030, d=$ 0.48 ; contrast estimate $(\mathrm{DBD} / \mathrm{CU}-\mathrm{vs}$ controls $)=43.18, p=0.013$, $d=0.52$, respectively. No significant difference emerged between DBD subgroups, contrast estimate $=4.24, p=0.82$, $d=0.04$.

Heart Rate ANOVAs revealed a significant difference between groups in HR responses to sadness, $F(2,60)=$ $3.31, p=0.043, \eta_{\mathrm{p}}^{2}=0.10$, but no significant difference during happiness $F(2,60)=1.94, p=0.153, \eta_{\mathrm{p}}^{2}=0.06$. An ANCOVA, with medication as a covariate, revealed no significant difference between groups in $\mathrm{HR}$ responses during anger, $F(2,59)=1.99, p=0.146, \eta_{\mathrm{p}}^{2}=0.06$. As to sadness, in agreement with predictions, follow-up $t$-tests demonstrated that the DBD/CU+ group showed significantly less HR deceleration during sadness than both the DBD/CU-, $t$ $(29)=-2.47, p=0.009$, one-tailed, $d=0.93$, and the control group, $t(44)=-2.21, p=0.016$, one-tailed, $d=0.79$ (see Fig. 1d). No significant difference emerged between DBD/ CU- and controls, $t(47)=0.93, p=0.356, d=0.26$.

Finally, within the DBD group stepwise regression analyses were conducted to examine the degree to which each of the empathy-related responses contributes to the prediction of the CU or I/CP factors. Medication was included as a dummy variable. The variables that significantly contributed to the model with outcome variable CU were: zygomaticus EMG reactivity during anger $(\beta=0.40, t=2.66, p=0.013)$, zygomaticus EMG reactivity during sadness $(\beta=-0.47, t=-2.99$, $p=0.006)$, and HR reactivity during sadness $(\beta=0.39, t=$ $2.43, p=0.022), F(3,27)=6.61, p=0.002$. The $R^{2}$ value for $\mathrm{CU}$ was 0.423 , indicating that the model accounted for $42 \%$ of the variability of the CU scores. No variables significantly contributed to the prediction of the $\mathrm{I} / \mathrm{CP}$ factor.

\section{Cardiovascular Function at Rest}

Resting HR (see Table 1) was not significantly different between DBD subgroups and controls, $F(2,60)=1.17$, $p=0.316, \eta_{\mathrm{p}}^{2}=0.04$. However, a significant difference was obtained in resting RSA expressed as proportional units,
$F(2,60)=3.20, p=0.048, \quad \eta_{\mathrm{p}}^{2}=0.10$. Follow-up $t$-tests revealed a significant difference between $\mathrm{DBD} / \mathrm{CU}+$ and controls, $t(44)=2.41, p=0.020, d=0.83$, and a marginally significant difference between both DBD subgroups, $t(29)=$ 2.05, $p=0.050, d=0.81$. No significant difference emerged between DBD/CU- and controls, $t(47)=.31, p=0.758, d=$ 0.07. Essentially the same results were obtained when expressing RSA as logarithmically transformed spectral power (see Table 1). As seen in Table 1, lower levels of resting RSA were obtained for DBD adolescents with high CU traits than for those with low CU traits or healthy controls. These differences could not be attributed to differences in respiratory frequency as estimates of peak respiratory frequency did not differ between groups, $F(2,60)=0.35$, $n s$.

\section{Discussion}

The main goal of this study was to examine emotional empathy in subgroups of DBD male adolescents and healthy controls. To our knowledge, this is the first study that examined autonomic activity together with verbal and facial EMG responses to different target emotions in DBD adolescents with high versus low CU traits. The study produced some interesting findings. First, in agreement with expectations, DBD adolescents with high CU traits showed significantly lower levels of empathic sadness than healthy controls across all response systems. Specifically, high CU respondents reported less empathy, showed less facial responsiveness, and less HR change from baseline during sadness than controls. Different from expectations, high CU respondents also reported less empathic happiness than controls. Second, consistent with conceptualizations of $\mathrm{CU}$ traits, the $\mathrm{CU}$ (not I/CP) factor was associated with empathy-related responses, including autonomic reactivity during sadness. Third, autonomic (not verbal nor facial) reactions to sadness produced distinct differences between DBD subgroups. In agreement with expectations, DBD adolescents with high $\mathrm{CU}$ traits showed significantly less HR change from baseline during sadness than those with low CU traits. Our findings are consistent with earlier findings demonstrating reduced autonomic response patterns during distress cue pictures (Blair 1999) and empathy-inducing film clips involving fear (Anastassiou-Hadjicharalambous and Warden 2008b) in antisocial children with high CU traits relative to those with low CU traits or controls. The results have been interpreted within Blair's $(1995,2006)$ Violence Inhibition Mechanism (VIM) model, which posits that reduced autonomic responses to distress cues may result from deficits within the VIM. The VIM is thought to be an innate mechanism for the control of aggression, typically activated by the sad and fearful expressions of others. 
Activation of the VIM results in autonomic arousal and the interruption of on-going (aggressive) behavior. Deficits within the VIM - resulting from a more general amygdala dysfunction - may lead to the development of aggressive behavior particularly seen in psychopathic individuals. Results of the current study are consistent with the VIM model by showing subnormal levels of HR reactivity during sadness in adolescents with high $\mathrm{CU}$ traits.

Heart rate reactivity reflects sympathetic and parasympathetic activation mediated by limbic and prefrontal structures, including the anterior cingulate cortex (ACC) (Bush et al. 2000; Devinsky et al. 1995; Medford and Critchley 2010; Thayer and Lane 2000). ACC is connected to anterior insula and both structures are involved in understanding emotional experiences of others (Singer et al. 2009), for example, in generating empathy when seeing someone in pain (Gallese et al. 2004). Reduced ACC responses to negative affective pictures have been observed in conduct disordered adolescents compared to controls (Stadler et al. 2007; Sterzer et al. 2005). Ventral and rostral areas of ACC have affective functions and are involved in the monitoring and regulation of social emotional responses (Adolphs 2003). Structural or functional changes in rostral ACC combined with changes in orbitofrontal cortex may cause prominent changes in social behavior and may lead to antisocial responses (Devinsky et al. 1995). An increased concentration of grey matter in medial orbitofrontal cortex, and in rostral and dorsal ACC has been observed in boys with conduct disorder and $\mathrm{CU}$ traits compared to healthy controls (de Brito et al. 2009), suggesting a delayed maturation of cortical areas implicated in processing of affective stimuli and the experience of empathy. Hence, subnormal HR reactivity to sadness by DBD adolescents with high CU traits may possibly relate to abnormalities in prefrontal brain regions including the ACC.

The current group of DBD adolescents with low CU traits showed a more diffuse pattern of empathy dysfunction. Relative to controls, they reported less empathic happiness, and showed deficits in facial responsiveness to both sadness and happiness. No impairments were observed in HR measures of empathic sensitivity. The data are not completely consistent with previous work (de Wied et al. 2005, 2009) suggesting that DBD boys are selectively impaired in empathy with negative (not positive) emotions. One possible explanation for the inconsistency is that DBD adolescents constitute a more heterogeneous group than DBD children because an older group is more likely to include both persons with adolescence-limited antisocial behavior and persons with early-onset pathways (Moffitt 1993). Alternatively, the variability of results could be due to differences in stimulus materials and/or developmental processes. For example, increasing testosterone levels in males at puberty and/or age-related changes in frontal lobe maturation are likely to affect empathic behavior (e.g., Decety 2010; Hermans et al. 2006).

\section{Basal Autonomic Function}

A second goal of the present study was to examine basal autonomic function in subgroups of DBD adolescents and controls. Resting HR was not significantly different between the three groups but resting RSA was significantly lower in DBD adolescents with high CU traits than in controls. Also, resting RSA tended to be lower in DBD adolescents with high $\mathrm{CU}$ traits compared to those with low CU traits. Since the three groups did not significantly differ in peak respiratory frequency, we may conclude that the observed differences in RSA level cannot be attributed to differences in respiratory frequency. This suggests that the high $\mathrm{CU}$ group was characterized by an abnormally low cardiac vagal tone. Although reduced RSA has been repeatedly observed in children or adolescents with DBD (Beauchaine 2001; Beauchaine et al. 2001, 2007, 2008; de Wied et al. 2009; Mezzacappa et al. 1997), a specific relationship with CU traits has not earlier been reported. It is important to mention in this connection that RSA scores were found to be inversely related to $\mathrm{CU}$ scores within the DBD group $(r=-40, p=0.026)$. Similar to reduced HR reactivity, reduced resting RSA in boys with $\mathrm{CU}$ traits may be an expression of structural or functional changes in brain circuits involving the ACC.

\section{Limitations and Strengths}

Several limitations of this study should be noted. First, the DBD sample was relatively small which makes it difficult to demonstrate significant differences between subgroups of DBD adolescents due to power limitations. Second, because the current study included only male adolescents, the findings require replication with female samples before they can be generalized to female adolescents with DBD. Third, because a majority of the DBD group showed comorbid ADHD, we cannot tell whether the results are exemplary for adolescents with pure $\mathrm{DBD}$, or comorbid $\mathrm{DBD} / \mathrm{ADHD}$. ADHD is related to DBD (Matthys and Lochman 2010) and therefore redundant as adjustment variable. Further research is needed to examine the independent effects of DBD and ADHD upon empathic behavior.

Strengths of our study can be seen in the inclusion of a well-defined sample of DBD adolescents, with high CU respondents (i.e., those with $\mathrm{CU}$ scores well above average) showing significantly higher levels of aggressive/rulebreaking behavior than low $\mathrm{CU}$ respondents. A further strength is the inclusion of positive and negative target emotions. Our results confirm that the nature of target 
emotions affects empathy-related responding in DBD adolescents. The findings encourage further research on empathy problems associated with DBD in relation to a broader range of target emotions, especially fear and anxiety.

\section{Clinical Implications}

If replicated, the findings may have clinical significance. In clinical practice it is important to discriminate conduct disordered children with CU traits from those without such traits because they may require different approaches to intervention. Our findings demonstrate distinct deficits in the autonomic (not verbal or facial) expression of empathic sadness across DBD subgroups, suggesting that the mechanisms underlying empathy problems may be different for those with high versus low CU traits. The multidimensional study of empathy may advance our understanding about the mechanisms underlying empathy problems in subgroups of DBD children and adolescents. If laboratory markers of empathy can be developed that distinguish between subtypes of DBD individuals (as autonomic indexes of empathy), such instruments could be quite useful as part of an assessment battery.

Broadening our understanding about the processes underlying deficits in the development of empathy proposed for DBD children and adolescents is likely to inform treatment options. Empathy training is often part of prevention and intervention programs for antisocial youth, as the EQUIP program (Gibbs 2003). Knowledge about the nature of empathy problems associated with DBD may have important implications for developing more individualized training programs aimed to strengthen empathic skills in DBD children and adolescents.

In sum, the data confirm that $\mathrm{CU}$ traits designate a subgroup of DBD adolescents who exhibit particularly high levels of aggression and delinquency. The data demonstrate that DBD adolescents with high CU traits show consistent impairments in empathic sadness across different response systems. Moreover, relative to those with low CU traits, DBD adolescents with high CU traits show reduced HR change from baseline while witnessing another person in distress. The data also demonstrate that DBD adolescents with high CU traits show subnormal tonic parasympathetic activation of the cardiac system (reflected by reduced RSA). Our findings support the notion that $\mathrm{CU}$ traits identify a distinct subgroup of DBD individuals, which is in line with the suggestion to include $\mathrm{CU}$ traits as a qualifier for CD in the DSM-V (e.g., Frick and Moffit 2010).

Acknowledgments We thank Annemarie Huiberts and Hilde van der Heu for assistance with data collection. We thank Bruce Gray for film editing, and Ton Aalbers for the development of the computerized empathy task and data analysis software.
Open Access This article is distributed under the terms of the Creative Commons Attribution Noncommercial License which permits any noncommercial use, distribution, and reproduction in any medium, provided the original author(s) and source are credited.

\section{References}

Achenbach, T. M. (1991a). Manual for the child behavior checklist and 1991 profile. Burlington: University of Vermont, Department of Psychiatry.

Achenbach, T. M. (1991b). Manual for the teacher's report form and 1991 profile. Burlington: University of Vermont, Department of Psychiatry.

Adolphs, R. (2003). Cognitive neuroscience of human social behaviour. Nature Reviews Neuroscience, 4, 165-178.

American Psychiatric Association. (2000). Diagnostic and statistical manual of mental disorders (DSM-IV-TR, 4th ed., text revision). Washington: American Psychiatric.

Anastassiou-Hadjicharalambous, X., \& Warden, D. (2008a). Cognitive and affective perspective-taking in conduct-disordered children high and low on callous-unemotional traits. Child and Adolescent Psychiatry and Mental Health, 2, 1-11.

Anastassiou-Hadjicharalambous, X., \& Warden, D. (2008b). Physiologically-indexed and self-perceived affective empathy in conduct-disordered children high and low on callousunemotional traits. Child Psychiatry and Human Development, 39, 503-517.

Beauchaine, T. P. (2001). Vagal tone, development, and Gray's motivational theory: toward an integrated model of autonomic nervous system functioning in psychopathology. Development and Psychopathology, 13, 183-214.

Beauchaine, T. P., Katkin, E. S., Strassberg, Z., \& Snarr, J. (2001). Disinhibitory psychopathology in male adolescents: discriminating conduct disorder from attention-deficit/hyperactivity disorder through concurrent assessment of multiple autonomic states. Journal of Abnormal Psychology, 110, 610-624.

Beauchaine, T. P., Gatzke-Kopp, L., \& Mead, H. K. (2007). Polyvagal theory and developmental psychopathology: emotion dysregulation and conduct problems from preschool to adolescence. Biological Psychology, 74, 174-184.

Beauchaine, T. P., Hong, J., \& Marsh, P. (2008). Sex differences in autonomic correlates of conduct problems and aggression. Journal of the American Academy of Child and Adolescent Psychiatry, 47, 788-796.

Berntson, G. G., Bigger, J. T., Jr., Eckberg, D. L., Grossman, P., Kaufmann, P. G., Malik, M., et al. (1997). Heart rate variability: origins, methods, and interpretive caveats. Psychophysiology, 34, 623-648.

Blair, R. J. R. (1995). A cognitive developmental approach to morality: investigating the psychopath. Cognition, 57, 1-29.

Blair, R. J. R. (1999). Responsiveness to distress cues in the child with psychopathic tendencies. Personality and Individual Differences, $27,135-145$.

Blair, R. J. R. (2005). Responding to the emotions of others: dissociating forms of empathy through the study of typical and psychiatric populations. Consciousness and Cognition, 14, 698718.

Blair, R. J. R. (2006). The emergence of psychopathy: implications for the neuropsychological approach to developmental disorders. Cognition, 1010, 414-442.

Blair, R. J. R. (2007). Empathic dysfunction in psychopathic individuals. In T. F. D. Farrow \& P. W. R. Woodruff (Eds.), Empathy in mental illness (pp. 3-16). New York: Cambridge University Press. 
Blair, R. J. R., Colledge, E., Murray, L., \& Mitchell, D. G. V. (2001). A selective impairment in the processing of sad and fearful expressions in children with psychopathic tendencies. Journal of Abnormal Child Psychology, 29, 491-498.

Burke, J. D., Loeber, R., \& Lahey, B. B. (2007). Adolescent conduct disorder and interpersonal callousness as predictors of psychopathy in young adults. Journal of Clinical Child and Adolescent Psychology, 36, 334-346.

Bush, G., Luu, P., \& Posner, M. I. (2000). Cognitive and emotional influences in anterior cingulate cortex. Trends in Cognitive Sciences, 4, 215-222.

Christian, R. E., Frick, P. J., Hill, N. L., Tyler, L., \& Frazer, D. R. (1997). Psychopathy and conduct problems in children: II. Implications for subtyping children with conduct problems. Journal of the American Academy of Child and Adolescent Psychiatry, 36, 233-241.

Cole, P. M., Zahn-Waxler, C., Fox, N. A., Usher, B. A., \& Welsh, J. D. (1996). Individual differences in emotion regulation and behavior problems in preschool children. Journal of Abnormal Psychology, $105,518-529$.

Dadds, M. A., Perry, Y., Hawes, D. J., Merz, S., Riddell, A. C., Haines, D. J., et al. (2006). Attention to the eyes and fear-recognition deficits in child psychopathy. The British Journal of Psychiatry, 189, 280-281.

Dadds, M. R., El Masry, Y., Wimalaweera, S., \& Guastella, A. J. (2008). Reduced eye gaze explains "fear blindness" in childhood psychopathic traits. Journal of the American Academy of Child and Adolescent Psychiatry, 47, 455-463.

Dadds, M. R., Jambrak, J., Pasalich, D., Hawes, D. J., \& Brennan, J. (2011). Impaired attention to the eyes of attachment figures and the developmental origins of psychopathy. Journal of Child Psychology and Psychiatry, 52, 238-245.

Davis, M. H. (1996). Empathy: A social psychological approach (1994, reprinted 1996). Social Psychology Series. Boulder: Westview.

de Brito, S. A., Mechelli, A., Wilke, M., Laurens, K. R., Jones, A. P., Barker, G. J., et al. (2009). Size matters: increased grey matter in boys with conduct problems and callous-unemotional traits. Brain, 132, 843-852.

de Wied, M., Goudena, P. P., \& Matthys, W. (2005). Empathy in boys with disruptive behavior disorders. Journal of Child Psychology and Psychiatry, 46, 867-880.

de Wied, M., van Boxtel, A., Posthumus, J. A., Goudena, P. P., \& Matthys, W. (2009). Facial EMG and heart rate responses to emotion-inducing film clips in boys with disruptive behavior disorders. Psychophysiology, 46, 996-1004.

de Wied, M., Gispen-de Wied, C. C., \& van Boxtel, A. (2010). Empathy dysfunction in children and adolescents with disruptive behavior disorders. European Journal of Pharmacology, 626, 97 103.

Decety, J. (2010). The neurodevelopment of empathy in humans. Developmental Neuroscience, 32, 257-267.

Denver, J. W., Reed, S. F., \& Porges, S. W. (2007). Methodological issues in the quantification of respiratory sinus arrhythmia. Biological Psychology, 74, 286-294.

Devinsky, O., Morrell, M. J., \& Vogt, B. A. (1995). Contributions of anterior cingulate cortex to behaviour. Brain, 118, 279-306.

Dimberg, U. (1990). Facial electromyography and emotional reactions. Psychophysiology, 27, 481-494.

Eisenberg, N., \& Eggum, N. D. (2009). Empathic responding: Sympathy and personal distress. In J. Decety \& W. Ickes (Eds.), The social neuroscience of empathy (pp. 71-83). Cambridge: MIT Press.

Eisenberg, N., \& Fabes, R. A. (1990). Empathy: conceptualization, measurement, and relation to prosocial behavior. Motivativation and Emotion, 14, 131-149.
Eisenberg, N., Losoya, S., Fabes, R. A., Guthrie, I. K., Reiser, M., Murphy, B., et al. (2001). Parental socialization of children's dysregulated expression of emotion and externalizing problems. Journal of Family Psychology, 15, 183-205.

Fairchild, G., van Goozen, S. H. M., Calder, A. J., Stollery, S. J., \& Goodyer, I. (2009). Deficits in facial expression recognition in male adolescents with early-onset or adolescence-onset conduct disorder. Journal of Child Psychology and Psychiatry, 50, 627636.

Ferdinand, R. F., \& van der Ende, J. (2002). NIMH-DISC-IV: Diagnostic interview schedule for children (Authorized Dutch version). Rotterdam: Erasmus $\mathrm{MC} /$ Sophia Kinderziekenhuis Rotterdam.

Ford, T., Goodman, R., \& Meltzer, H. (2003). The British child and adolescent mental health survey 1999: the prevalence of DSM-IV disorders. Journal of the American Academy of Child and Adolescent Psychiatry, 42, 1203-1211.

Frick, P. J., \& Hare, R. D. (2001). Antisocial process screening device (APSD). Toronto: Multi-Health Systems.

Frick, P. J., \& Moffit, T. E. (2010). A proposal to the DSM-V childhood disorders and the ADHD and disruptive behavior disorders work groups to include a specifier to the diagnosis of conduct disorder based on the presence of callous-unemotional traits (pp. 1-36). USA: American Psychiatric Association.

Frick, P. J., \& White, S. F. (2008). Research review: the importance of callous-unemotional traits for the developmental models of aggressive and antisocial behavior. The Journal of Child Psychology and Psychiatry, 49, 359-375.

Frick, P. J., Cornell, A. H., Barry, C. T., Bodin, S. D., \& Dane, H. E. (2003). Callous-unemotional traits and conduct problems in the prediction of conduct problem severity, aggression, and selfreport of delinquency. Journal of Abnormal Child Psychology, $31,457-470$.

Frick, P. J., Stickle, T. R., Dandreaux, D. M., Farrell, J. M., \& Kimonis, E. R. (2005). Callous-unemotional traits in predicting the severity and stability of conduct problems and delinquency. Journal of Abnormal Child Psychology, 33, 471-487.

Gallese, V., Keysers, C., \& Rizzolatti, G. (2004). A unifying view of the basis of social cognition. Trends in Cognitive Sciences, 8 , 396-403.

Gibbs, J. C. (2003). Moral development \& reality: Beyond the theories of Kohlberg and Hoffman. London: Sage.

Grossman, P., \& Kollai, M. (1993). Respiratory sinus arrhythmia, cardiac vagal tone, and respiration: within- and betweenindividual relations. Psychophysiology, 30, 486-495.

Grossman, P., \& Taylor, E. W. (2007). Toward understanding respiratory sinus arrhythmia: relations to cardiac vagal tone, evolution and biobehavioral functions. Biological Psychology, 74, 263-285.

Hermans, E. J., Putman, P., \& van Honk, J. (2006). Testosterone administration reduces empathic behavior: a facial mimicry study. Psychoendocrinology, 31, 859-866.

Hoffman, M. L. (2008). Empathy and prosocial behavior. In M. Lewis, J. M. Haviland-Jones, \& L. Feldman Barrett (Eds.), Handbook of emotions (pp. 440-455). New York: Guilford.

Jones, A. P., Laurens, K. R., Herba, C. M., Barker, G. J., \& Viding, E. (2009). Amygdala hypoactivity to fearful faces in boys with conduct problems and callous-unemotional traits. The American Journal of Psychiatry, 166, 95-102.

Jones, A. P., Happé, F. G. E., Gilbert, F., Burnett, S., \& Viding, E. (2010). Feeling, caring, knowing: different types of empathy deficit in boys with psychopathic tendencies and autism spectrum disorder. Journal of Child Psychology and Psychiatry, 51, 11881197.

Kreibig, S. D., Wilhelm, F. H., Roth, W. T., \& Gross, J. J. (2007). Cardiovascular, electrodermal, and respiratory response patterns 
to fear- and sadness-inducing films. Psychophysiology, 44, 787806.

Larsen, J. T., Norris, C. J., \& Cacioppo, J. T. (2003). Effects of positive and negative affect on electromyographic activity over zygomaticus major and corrugator supercilii. Psychophysiology, 40, 776-785.

Lorber, M. F. (2004). Psychophysiology of aggression, psychopathy, and conduct problems: a meta-analysis. Psychological Bulletin, $130,531-552$.

Lovett, B. J., \& Sheffield, R. A. (2007). Affective empathy deficits in aggressive children and adolescents: a critical review. Clinical Psychology Review, 27, 1-13.

Lundqvist, L.-O., \& Dimberg, U. (1995). Facial expressions are contagious. Journal of Psychophysiology, 9, 203-211.

Marsh, A. A., Finger, E. C., Mitchell, D. G. V., Reid, M. E., Sims, C., Kosson, D. S., et al. (2008). Reduced amygdala response to fearful expressions in children and adolescents with callousunemotional traits and disruptive behavior disorders. The American Journal of Psychiatry, 165, 712-720.

Matthys, W., \& Lochman, J. E. (2010). Oppositional defiant disorder and conduct disorder in childhood. Oxford: Wiley-Blackwell.

Medford, N., \& Critchley, H. D. (2010). Conjoint activity of anterior insular and anterior cingulate cortex: awareness and response. Brain Structure \& Function, 214, 535-549.

Mezzacappa, E., Tremblay, R. E., Kindlon, D., Saul, J. P., Arseneault, L., Seguin, J., et al. (1997). Anxiety, antisocial behavior, and heart rate regulation in adolescent males. Journal of Child Psychology and Psychiatry, 38, 457-469.

Miller, P. A., \& Eisenberg, N. (1988). The relation of empathy to aggressive and externalizing/antisocial behavior. Psychological Bulletin, 103, 324-344.

Moffitt, T. E. (1993). Adolescence-limited and life-course-persistent antisocial behavior: a developmental taxonomy. Psychological Review, 100, 674-701.

Mulder, L. J. M., Hofstetter, H., \& van Roon, A. (2007). CARSPAN for windows: User's manual. Groningen: University of Groningen.

Olsson, A., \& Phelps, E. A. (2007). Social learning of fear. Nature Neuroscience, 10, 1095-1102.

Ortiz, J. O., \& Raine, A. (2004). Heart rate level and antisocial behavior in children and adolescents: a meta-analysis. Journal of the American Academy of Child and Adolescent Psychiatry, 43, $154-162$.

Pardini, D. A., \& Fite, A. J. (2010). Symptoms of conduct disorder, oppositional defiant disorder, attention-deficit/hyperactivity disorder, and callous-unemotional traits as unique predictors of psychosocial maladjustment in boys: advancing an evidence base for DSM-V. Journal of the American Academy of Child and Adolescent Psychiatry, 49, 1134-1144.

Piferi, R. L., Kline, K. A., Younger, J., \& Lawler, K. A. (2000). An alternative approach for achieving cardiovascular baseline: viewing an aquatic video. International Journal of Psychophysiology, 37, 207-217.

Raine, A. (2002). Annotation: the role of prefrontal deficits, low autonomic arousal, and early health factors in the development of antisocial and aggressive behavior in children. Journal of Child Psychology and Psychiatry, 43, 417-434.

Ritz, T., \& Dahme, B. (2006). Implementation and interpretation of respiratory sinus arrhythmia measures in psychosomatic medicine: practice against better evidence? Psychosomatic Medicine, $68,617-627$.

Sattler, J. M. (1992). Assessment of children (3rd ed.). San Diego: Jerome M. Sattler.

Scheepers, F. E., Buitelaar, J. K., \& Matthys, W. (2011). Conduct disorder and the specifier callous and unemotional traits in the DSM-5. European Journal of Child and Adolescent Psychiatry, 20, 89-93.
Singer, T., Critchley, H. D., \& Preuschoff, K. (2009). A common role of insula in feelings, empathy and uncertainty. Trends in Cognitive Sciences, 13, 334-340.

Sonnby-Borgström, M. (2002). Automatic mimicry reactions as related to differences in emotional empathy. Scandinavian Journal of Psychology, 43, 433-443.

Sonnby-Borgström, M., Jönsson, P., \& Svensson, O. (2003). Emotional empathy as related to mimicry reactions at different levels of information processing. Journal of Nonverbal Behavior, 27, 3-23.

Stadler, C., Sterzer, P., Schmeck, K., Krebs, A., Kleinschmidt, A., \& Poustka, F. (2007). Reduced anterior cingulate activation in aggressive children and adolescents during affective stimulation: association with temperament traits. Journal of Psychiatric Research, 41, 410-417.

Sterzer, P., Stadler, C., Krebs, A., Kleinschmidt, A., \& Poustka, F. (2005). Abnormal neural responses to emotional visual stimuli in adolescents with conduct disorder. Biological Psychiatry, 57, 7-15.

Stevens, D., Charman, T., \& Blair, R. J. R. (2001). Recognition of emotion in facial expressions and vocal tones in children with psychopathic tendencies. The Journal of Genetic Psychology, 162, 201-211.

Task Force of the European Society of Cardiology and the North American Society of Pacing and Electrophysiology. (1996). Heart rate variability. Standards of measurement, physiological interpretation, and clinical use. Circulation, 93, 1043-1065.

Thayer, J. F., \& Lane, R. D. (2000). A model of neurovisceral integration in emotion regulation and dysregulation. Journal of Affective Disorders, 61, 201-216.

Thayer, J. F., Sollers, J. J., III, Ruiz-Padial, E., \& Vila, J. (2002). Estimating respiratory frequency from autoregressive spectral analysis of heart period. IEEE Engineering in Medicine and Biology, 21, 41-45.

Tsai, J. L., Levenson, R. M., \& Carstensen, L. L. (2000). Autonomic, subjective, and expressive responses to emotional films in older and younger Chinese Americans and European Americans. Psychology and Aging, 15, 684-693.

Vaughn, M. G., Howard, M. O., \& DeLisi, M. (2008). Psychopathic personality traits and delinquent careers: an empirical examination. International Journal of Law and Psychiatry, 31, 407-416.

Verhulst, F. C., van der Ende, J., Ferdinand, R. F., \& Kasius, M. C. (1997). The prevalence of DSM-III-R diagnoses in a national sample of Dutch adolescents. Archives of General Psychiatry, 54, 329-336.

Viding, E., Blair, R. J. R., Moffitt, T. E., \& Plomin, R. (2005). Evidence for substantial genetic risk for psychopathy in 7-yearolds. Journal of Child Psychology and Psychiatry, 46, 592-597.

Waldstein, S. R., Kop, W. J., Schmidt, L. A., Haufler, A. J., Krantz, D. S., \& Fox, N. A. (2000). Frontal electrocortical and cardiovascular reactivity during happiness and anger. Biological Psychology, 55, 3-23.

Wechsler, D. (1974). Manual for the Wechsler Intelligence Scale for Children-revised. New York: Psychological Corporation.

Westbury, H. R., \& Neumann, D. L. (2008). Empathy-related responses to moving film stimuli depicting human and nonhuman animal targets in negative circumstances. Biological Psychology, 78, 66-74.

Zahn, T. P., \& Kruesi, M. J. P. (1993). Autonomic activity in boys with disruptive behavior disorders. Psychophysiology, 30, 605-614.

Zhou, Q., Eisenberg, N., Losoya, S. H., Fabes, R. A., Reiser, M., Guthrie, I. K., et al. (2002). The relations of parental warmth and positive expressiveness to children's empathy-related responding and social functioning: a longitudinal study. Child Development, 73, 893-915.

Zhou, Q., Valiente, C., \& Eisenberg, N. (2003). Empathy and its measurement. In S. J. Lopez \& C. R. Snyder (Eds.), Positive psychological assessment: A handbook of models and measures (pp. 269-284). Washington: American Psychological Association. 